\title{
Stellar Populations of Decoupled Cores in E/S0 Galaxies with SAURON and OASIS
}

\section{Richard M. McDermid ${ }^{1}$, Eric Emsellem ${ }^{2}$, Kristen L. Shapiro ${ }^{3}$, Roland Bacon $^{2}$, Martin Bureau ${ }^{4}$, Michele Cappellari ${ }^{1,4}$, Roger L. Davies ${ }^{4}$, Tim de Zeeuw ${ }^{1}$, Jesús Falcón-Barroso ${ }^{1,5}$, Davor Krajnović $^{4}$, Harald Kuntschner ${ }^{6}$, Reynier F. Peletier ${ }^{7}$ and Marc Sarzi ${ }^{8}$}

\author{
${ }^{1}$ Leiden Observatory, Leiden University, Postbus 9513, 2300 RA Leiden, The Netherlands \\ email: mcdermid@strw.leidenuniv.nl \\ ${ }^{2}$ Université de Lyon 1, CRAL, Observatoire de Lyon, 9 av. Charles André, F-69230 \\ Saint-Genis Laval; CNRS, UMR 5574 ; ENS de Lyon, France \\ ${ }^{3}$ UC Berkeley Department of Astronomy, Berkeley, CA 94720, USA \\ ${ }^{4}$ Denys Wilkinson Building, University of Oxford, Keble Road, Oxford, United Kingdom \\ ${ }^{5}$ European Space and Technology Centre (ESTEC), Keplerlaan 1, Postbus 299, 2200 AG \\ Noordwijk, The Netherlands \\ ${ }^{6}$ Space Telescope European Coordinating Facility, European Southern Observatory, \\ Karl-Schwarzschild-Str 2, 85748 Garching, Germany \\ ${ }^{7}$ Kapteyn Astronomical Institute, Postbus 800, 9700 AV Groningen, The Netherlands \\ ${ }^{8}$ Centre for Astrophysics Research, Science \& Technology Research Institute, University of \\ Hertfordshire, Hatfield, United Kingdom
}

\begin{abstract}
We summarize results from McDermid et al. (2006), who present a set of followup observations of the SAURON representative survey of early-type galaxies. We used the OASIS integral-field spectrograph (while at the Canada-France-Hawaii Telescope) to obtain high spatial resolution spectra of 28 elliptical and lenticular galaxies. These seeing-limited data have on average twice the spatial resolution of the SAURON data, albeit over a smaller field. These new data reveal previously unresolved features in these objects' stellar kinematics, stellar populations, and ionized gas properties. In this contribution, we focus on the discovery of a population of compact kinematically decoupled cores in a number of our sample galaxies. These compact cores are related to regions of young stars, and counter-rotate around the host galaxy's minor axis. We compare these objects to previously known decoupled components, which in contrast are composed of old stars, and which rotate around axes unrelated to the host galaxy's kinematics or shape. A key difference between these two kinds of decoupled cores are their physical size and relative mass. The compact decoupled cores are smaller than a few hundred parsec, and constitute less than a few percent of the total galaxy mass. The 'classical' decoupled cores exist on kiloparsec scales, and comprise around a factor 10 more mass. We suggest that the small components are only found with young ages because of their low mass-to-light ratio. We show that after a few Gyrs, these components 'fade' into the background galaxy, making them more difficult to detect. We draw the following conclusions: 1) young stars found in early-type galaxies are very often associated with centrally-concentrated counter-rotating components; 2) the small mass fraction and kinematic decoupling of these cores suggests that the star formation is associated to minor accretion events, which effectively drive the spread in luminosity-weighted ages found in early-type galaxies; and 3) such decoupled components may be common in all earlytype galaxies, but not directly observed due to their small contribution to the total galaxy light at older ages.
\end{abstract}

Keywords. galaxies: elliptical and lenticular, cD; evolution; formation; kinematics and dynamics; stellar content; structure 


\section{Introduction}

We are carrying out a program of high spatial resolution integral-field spectroscopy of early-type galaxy nuclei. We select our sample from galaxies observed as part of the SAURON survey: a study of 72 representative nearby early-type galaxies and spiral bulges observed with SAURON, our custom-built panoramic integral-field spectrograph mounted at the William Herschel Telescope (WHT) on La Palma (Bacon et al. 2001). We have observed the centres of a subset of 28 galaxies from the SAURON sample using the OASIS integral-field spectrograph, mounted on the 3.6-m Canada-France-Hawaii Telescope (CFHT), Hawaii. OASIS provides good sampling of the natural seeing on Mauna Kea, resulting in twice the spatial resolution of the SAURON data on average. Maps for all measured quantities (stellar kinematics, ionised gas fluxes and kinematics, Lick indices and stellar population parameters) are presented in McDermid et al. (2006).

\section{Analysis: Deriving the Stellar Population Parameters}

The spectral range of our OASIS data contains a number of absorption features which can be used as diagnostic tools to determine the distribution of stellar populations within a galaxy, in terms of the luminosity-weighted population parameters: age, metallicity and element abundance ratio. We quantify the depth of these absorption features through the use of line-strength indices, taken from the Lick/IDS system (e.g. Burstein et al. 1984). Three absorption features in our spectral range, namely $\mathrm{H} \beta$, Fe5015 and $\mathrm{Mg} b$, can be significantly altered due to 'infilling' by possible $\mathrm{H} \beta$, [OIII] and [NI] emission features, respectively. Before measuring the absorption-line strengths, we therefore subtract the emission spectrum from the original data, using the spectral-fitting technique of Sarzi et al. (2006).

In order to determine the stellar population parameters, we use the single-burst stellar population models of Thomas, Maraston \& Bender (2003). We oversample the original grid of models to reduce discretization effects, and locate the model which lies closest to our six measured Lick indices simultaneously, based on the value of $\chi^{2}$, similar to the method described in Proctor, Forbes, \& Beasley (2004).

\section{Results: Stellar Populations of Kinematically Decoupled Cores}

The SAURON representative sample contains numerous examples of kinematically decoupled components (KDCs), many of which are also in our present OASIS subsample. To these, we add three KDCs which are visible in our OASIS data, though not resolved with SAURON: NGC 3032, NGC 4382, and NGC 4621. The term KDC refers here to objects with subcomponents that either rotate around a different axis to the main galaxy, or that rotate around the same axis, but with an opposite sense of rotation ('counterrotating'). With this definition, we do not consider dynamically decoupled structures which co-rotate with the main galaxy, such as nuclear disks, even though there are several such structures in our sample. Misaligned and counter-rotating components offer the advantage of being clearly distinguished from the rest of the galaxy, and may indeed have a distinct formation process from the co-rotating case.

Figure 1 shows that intrinsically large $(\geqslant 1 \mathrm{kpc})$ KDCs have predominantly old $(\geqslant 10 \mathrm{Gyr})$ populations. The group of small $(<1 \mathrm{kpc})$ KDCs, by contrast, exhibits populations spanning the full range of ages, from 0.5 Gyr to $15 \mathrm{Gyr}$, with five of the six small KDCs younger than 5 Gyr. The colours used in this figure indicate the class of rotation, being either a 'fast-rotating' object, mainly supported by rotation (blue symbols); or a 'slow-rotating' object, mainly supported by dynamical pressure (red symbols). 


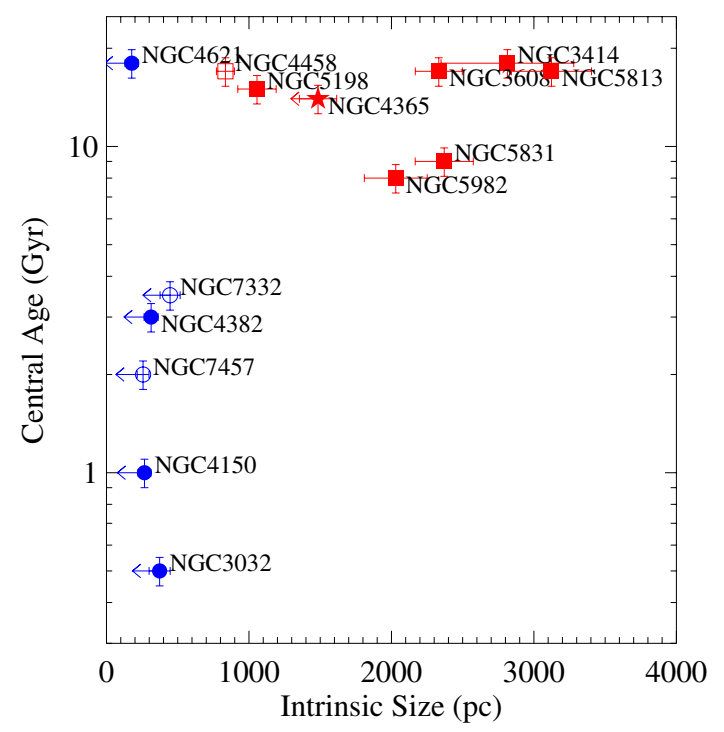

Figure 1. Intrinsic size of KDCs versus their central luminosity-weighted age. Closed symbols show objects observed by both SAURON and OASIS. Open symbols show objects only observed with SAURON. Red squares indicate slow-rotators and blue circles indicate fast rotators, as defined in Emsellem et al. (submitted). Arrows indicate where the measured diameter of the KDC is less than four times the seeing FWHM, implying that the KDC is not well-resolved and may be smaller than measured here.

There are clearly no examples of slow-rotating objects with young, compact KDCs. The age-distribution of fast-rotating galaxies with counter-rotation is biased towards objects with younger global luminosity-weighted mean ages. Five of the seven fast-rotating objects younger than $<5$ Gyr show a counter-rotating core. Only one of the remaining fast-rotating galaxies older than 5 Gyr shows a detectable KDC.

The higher occurrence of counter-rotation in young galaxies may simply be the effect of luminosity weighting. These small components contribute relatively little in mass to the galaxy, but are still visible above the background of the main body because their young age (and therefore low $\mathrm{M} / \mathrm{L}$ ) makes them very bright. We explore this with the following 'toy' model. Using the measured central line-strengths of NGC 4150, we constrain the amount of mass of young stars within the central aperture that can be added to a background 'base' population (assumed to be that of the outer parts of the galaxy). Our SAURON data indicate an age of $\sim 5$ Gyr for the outer body of this galaxy. The central $\mathrm{H} \beta$ value is $\sim 3.6 \AA$, allowing a mass fraction of around $6 \%$ of a 0.5 Gyr population to be added. Note that this is only the contribution within the $1^{\prime \prime}$ line-of-sight, not to the entire system.

We simulate a KDC velocity field using a polar Fourier expansion with ad hoc coefficient profiles, consisting of a large-scale rotating component, with a compact counterrotating component. We assign the two velocity fields to SSP model spectra of Bruzual \& Charlot (2003) of appropriate age, assigning the young population to the compact counter-rotating component, and combine the spectra using the corresponding massweighting. Noise is added to the simulated data cube, which is then spatially binned, and the kinematics are extracted using pPXF, consistent with how we treat our observational data. The result is a realistic-looking KDC velocity field (Figure 2).

We then hold the mass fraction fixed, and 'evolve' the populations in step by combining older SSP models with the same age difference. As the KDC population ages, its 

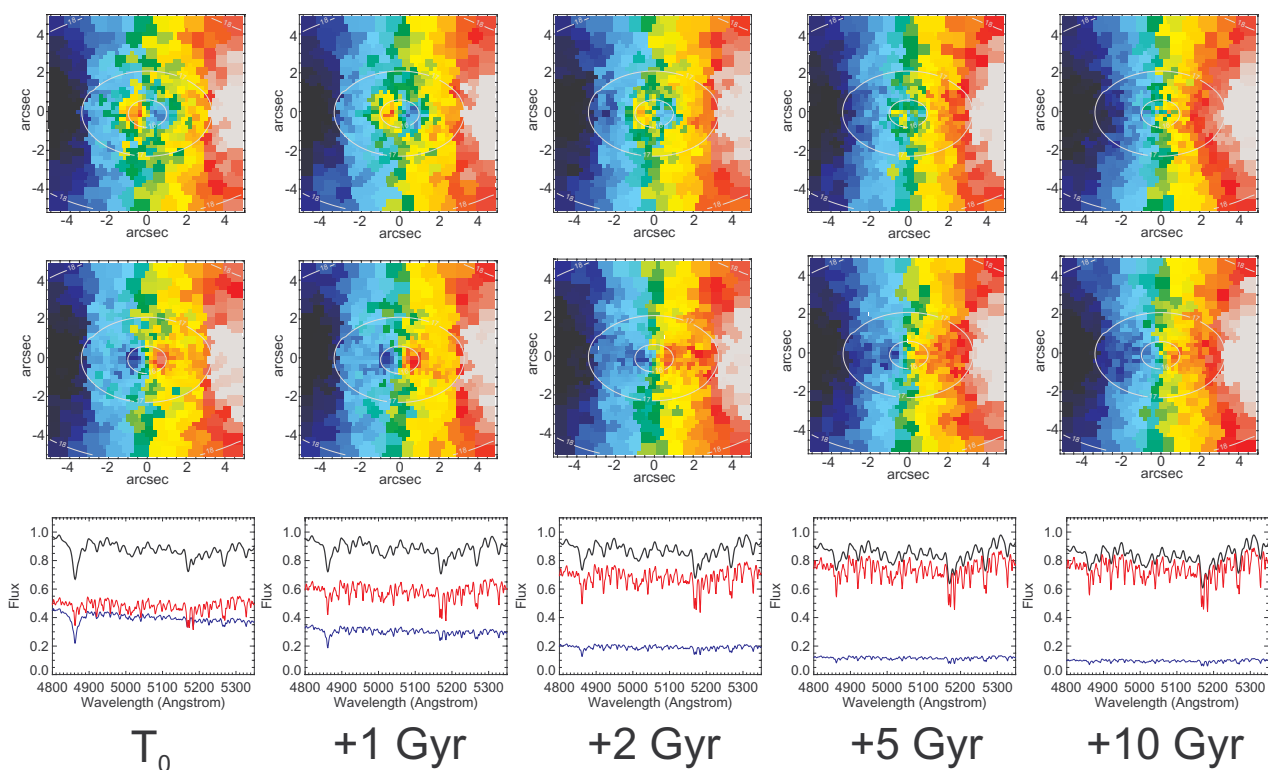

$\mathrm{T}_{0}$

+1 Gyr

$+2 \mathrm{Gyr}$

+5 Gyr

Figure 2. Model velocity fields for a counter-rotating (top row) and co-rotating (middle row) young subcomponent. The spectrum of the young component (blue - lower) and older main component (red - middle) are shown on the bottom row, indicating there luminosity-weighted contribution to the observed spectrum (black).

mass-to-light ratio increases, resulting in a dimming of the KDC stars. The effect is to 'fade' the KDC into the background rotation field. After 5 Gyr, the KDC is barely visible. This helps explain the apparent lack of intermediate- and old-aged small KDCs.

Assuming that young co- and counter-rotating components trace the angular momentum vector of 'seed' material which has recently entered the system from outside, one would expect that both types of component are equally likely to form. It is therefore somewhat surprising that five of the seven youngest galaxies in our full sample show evidence of counter-rotating components. It is tempting to conclude that counter-rotation is somehow connected to enhanced star-formation. These are, however, small-number statistics, and the significance that counter-rotating components tend to be young is not high.

The middle row of Figure 2 uses the same kinematic components and populations as the top row, but in this case the subcomponent is co-rotating. The impact of the co-rotating component on the total observed velocity field is clearly more subtle, visible only as an additional 'pinching' of the central velocity field. The co-rotating component becomes difficult to separate from the background field after only $\sim 2$ Gyr. For this reason, the intrinsic distribution of co- and counter-rotating central young components could actually be rather similar, but since the co-rotating cases can only be clearly identified at very young ages, our sample of young galaxies is currently too small to determine this reliably.

\section{Conclusions}

Considering all galaxies in the SAURON survey that show a clear kinematically decoupled stellar component, we explore the relationship between central stellar population and the size of the component. We find that, although the 'classical' decoupled components show rather old stellar populations, there also exists a population of compact $(\lesssim 100 \mathrm{pc})$ 
decoupled components which show a range of ages, from 15 Gyr to $\lesssim 0.5$ Gyr. Given the large difference in intrinsic size, we conclude that the two types of decoupled component are not directly related in the evolutionary sense, and that the compact variety are not necessarily an important feature by mass, although the young stars within these decoupled components are largely responsible for the significant spread in integrated luminosity-weighted ages observed for these galaxies.

Our data suggest a link between counter-rotation and enhanced star-formation. By consideration of a simple model, we conclude that older KDCs of similar mass fractions are rather difficult to detect, and so there may be a sizeable number of low-mass counterrotating components that we are missing. Likewise, co-rotating components of the same mass fraction leave a more subtle signature on the observed stellar kinematics, and so can only be detected when its population is rather young. Our sample is therefore too small to reliably put constraints on the specific importance of counter-rotation to recent star formation.

\section{Acknowledgements}

We thank the organizers for a timely and efficiently organized conference, and the participants for many enjoyable discussions and interactions, making this a very pleasant meeting.

\section{References}

Bacon, R., Copin, Y., Monnet, G., et al. 2001, MNRAS 326, 23

Bruzual, G., \& Charlot, S. 2003, MNRAS 344, 1000

Burstein, D., Faber, S. M., Gaskell, C. M., \& Krumm, N. 1984, ApJ 287, 586

McDermid, R. M., Emsellem, E., Shapiro, K. L., et al. 2006, MNRAS 373, 906

Proctor, R. N., Forbes, D. A., \& Beasley, M. A. 2004, MNRAS 355, 1327

Sarzi, M., et al. 2006, MNRAS 366, 1151

Thomas, D., Maraston, C., \& Bender, R. 2003, MNRAS 339, 897

\section{Discussion}

Cid FERnANDEs: I wonder if you have information on the emission-line properties of your galaxies. From work with Gonzalez Delgado and other friends, I would predict these counter-rotatin cores you find match what are called 'young-transition' objects; i.e., objects with substantial $\sim 1$ Gyr populations and $[\mathrm{OI} / \mathrm{H} \alpha]<0.25$.

MCDERMID: Indeed, these objects are transitional in many aspects, also from their morphologies, which tend to be closer to early-type bulges than E/S0. Unfortunately, we do not have sufficient emission-line diagnostics to fully understand the ionized gas component, but this may be the purpose of future observations.

BALCELLS: Do the massive KDCs show any form of metallicity decoupling? Do their core metallicities differ from those of other ellipticals without KDCs?

MCDERMID: We do not find any strong signatures of the KDCs in either the twodimensional distribution of metallicities or ages compared with those of other galaxies photometrically classified as elliptical. Something we are currently exploring is linking the populations more directly with the stellar kinematics of our complete early-type sample: ellipticals and lenticulars. We have already found that galaxies with strong rotation have an $\mathrm{Mg} b$ distribution more flattened than the isophotes, suggesting a connection with the disk component. We are not yet sure, however, where KDCs fit into this picture. 